Research Article

\title{
Prevalence of Hepatitis C Virus Infection among Pregnant Women in Ethiopia: A Systematic Review and Meta-Analysis
}

\author{
Birye Dessalegn Mekonnen (iD \\ Department of Nursing, Teda Health Science College, P.O. BOX 790, Gondar, Ethiopia \\ Correspondence should be addressed to Birye Dessalegn Mekonnen; birye22@gmail.com
}

Received 17 December 2020; Revised 12 February 2021; Accepted 20 May 2021; Published 28 May 2021

Academic Editor: Gerardo E. Guillen Nieto

Copyright ( 2021 Birye Dessalegn Mekonnen. This is an open access article distributed under the Creative Commons Attribution License, which permits unrestricted use, distribution, and reproduction in any medium, provided the original work is properly cited.

\begin{abstract}
Background. Hepatitis $\mathrm{C}$ virus infection during pregnancy is associated with a high risk of maternal complications and poor birth outcomes. There are variable reports on the prevalence of hepatitis $\mathrm{C}$ virus infection among pregnant women in Ethiopia. Therefore, this study aims to estimate the pooled prevalence of hepatitis $\mathrm{C}$ virus infection among pregnant women in Ethiopia. Methods. A comprehensive search of electronic databases including PubMed, Scopus, EMBASE, the Cochrane Library, Web of Sciences, and Google Scholar was conducted from April 03, 2020, to May 03, 2020. The quality of included article was evaluated by the JBI. Heterogeneity between the studies was assessed using Cochrane $Q$ and $I^{2}$ test. The presence of publication bias was tested by funnel plots and Egger's test. A random-effects meta-analysis was computed to determine the pooled prevalence of HCV infection among pregnant women. Results. Of 502 studies, 6 studies with a total of 2117 pregnant women were included in the meta-analysis. The overall pooled prevalence of hepatitis $C$ virus infection among pregnant women in Ethiopia was $1.83 \%(95 \%$ CI: 0.61, 3.06). Besides, subgroup analysis revealed that the highest HCV prevalence among pregnant women was observed in Oromia region, $5.10 \%(95 \% \mathrm{CI}:-0.53,10.73)$. Conclusions. This study shows an intermediate level of HCV infection among pregnant women in Ethiopia. The finding suggests the need of implementing a routine hepatitis $C$ virus screening program for all pregnant women, which enables women to access $\mathrm{HCV}$ antiviral treatment to minimize vertical transmission to the newborn infants. Moreover, national and regional health programs should mandate and monitor the screening procedures so as to reduce the risk of hepatitis $C$ virus infection.
\end{abstract}

\section{Background}

Hepatitis $\mathrm{C}$ infection is an emerging worldwide public health concern affecting millions of people each year $[1,2]$. Hepatitis $\mathrm{C}$ virus $(\mathrm{HCV})$ is the etiology of hepatitis $\mathrm{C}$ infection that can cause acute and chronic hepatitis and potentially lead to the development of cirrhosis, liver cancer, or death of infected patients $[2,3]$

According to World Health Organization (WHO) global health impact report of viral hepatitis, about 130 to 150 million people were affected by chronic HCV infection globally, reaching endemic proportions in sub-Saharan Africa (SSA). It is also estimated to result in 350,000 to 500,000 deaths annually [4]. The WHO estimates, about 3-4 million peoples are infected each year with most of the cases occurring in Africa [5, 6]. Another evidence also indicated that an estimated 71.1 million people are infected with $\mathrm{HCV}$ worldwide [3]. In most African countries, HCV remains underdiagnosed and underreported even it is highly infectious [7].

The prevalence of maternal $\mathrm{HCV}$ infection during pregnancy ranged from 1 to $8 \%$ worldwide [8]. Thus, the prevention of $\mathrm{HCV}$ vertical transmission is very important [9]. Like other routes such as intravenous drug use or blood product transfusion, transmission during sexual, $\mathrm{HCV}$ can be transmitted during delivery $[4,10]$. The transmission of HCV from mother to child ranged from 4 to $8 \%$ [9].

Maternal HCV infection during pregnancy is associated with a high risk of maternal complications including preterm delivery, placental separation, vaginal bleeding, 
premature rupture of membranes, and mortality $[11,12]$. It is also associated with a high risk of neonatal hepatitis that can lead to liver cirrhosis and hepatocellular carcinoma in young adults $[13,14]$. Furthermore, infants born to women with hepatitis $\mathrm{C}$ infection are at risk of poor birth outcomes, including low birth weight, preterm birth, and congenital anomaly [15].

In the health system of Ethiopia, the health burden due to viral hepatitis, in general, is still given less $[16,17]$. Though viral hepatitis screening is recommended during routine antenatal care (ANC), regular antenatal screening of pregnant women is not common and obligatory in Ethiopia [18]. Recent findings revealed that low awareness of the hepatitis virus was reported in both the general population and healthcare professionals $[16,19]$.

In the control of vertical transmission of HCV disease, estimating its prevalence among pregnant women is very important. There are variable reports on the prevalence of $\mathrm{HCV}$ infection among pregnant women in Ethiopia; however, the variations have not been examined systematically. Moreover, there was not a nationwide study assessing the pooled prevalence of $\mathrm{HCV}$ infection among pregnant women. This study aims to estimate the pooled prevalence of $\mathrm{HCV}$ infection among pregnant women based on the available studies using systematic review and meta-analysis methods. The findings of this meta-analysis will help policymakers and other concerned bodies to provide a quantified estimate of the problem as a step toward a better understanding of the HCV epidemiology, identify gaps in $\mathrm{HCV}$ screening during ANC, and plan strategies to increase awareness of the general population and healthcare workers regarding the epidemiology of $\mathrm{HCV}$.

\section{Methods}

2.1. Study Design and Setting. A systematic review and metaanalysis was conducted to estimate the prevalence of $\mathrm{HCV}$ during pregnancy in Ethiopia. This review was conducted in Ethiopia which is found in the Horn of Africa. The country covers an area of $1,100,000 \mathrm{~km}^{2}$ and divided into 9 regions, namely, Tigray, Afar, Amhara, Oromia, Somali, Benishangul-Gumuz, Southern Nations Nationalities and People Region (SNNPR), Gambella, Harari, and two Administrative states (Addis Ababa city administration and Dire Dawa city administration). Currently, the population of Ethiopia is estimated to be more than 112 million [20]. In Ethiopia, there are no established programs and strategies for the vaccination of children, pregnant women, health professionals, and the general population against $\mathrm{HCV}$ infection. Antenatal screening for $\mathrm{HCV}$ to all pregnant women and vaccination of their babies at birth is recommended.

2.2. Search Strategy and Information Sources. The search was focused on the prevalence of HCV during pregnancy in Ethiopia and carried out according to the Preferred Reporting Items for Systematic Reviews and Meta-Analyses (PRISMA) statement [21]. To find studies conducted on the prevalence of $\mathrm{HCV}$ infection among pregnant women, systematic literature searches were conducted on PubMed, Scopus, EMBASE, the Cochrane Library, Web of Sciences, Directory of Open Access Journal, and Google scholar. The search was conducted from April 03, 2020, to May 03, 2020. The search was carried out based on the following keywords: "prevalence", "epidemiology", "seroprevalence”, "hepatitis C Virus", "HCV", "hepacivirus", "hep C”, "pregnant women”, and "Ethiopia." The search terms were used independently and in combination using Boolean operators like "OR" or “AND” (supplementary file 1).

2.3. Study Selection. During the advanced search, articles/ documents were downloaded into Endnote software version $7 \times 2.1$ to manage references. After excluding duplicates, titles and abstracts and full texts of the remained papers were screened to determine the relevance of the studies. The PRISMA flow diagram was used to summarize the study selection processes [22].

\subsection{Inclusion and Exclusion Criteria}

\subsubsection{Inclusion Criteria.}

Study area: only studies conducted in Ethiopia were included

Design: all observational studies reporting the prevalence of $\mathrm{HCV}$ among pregnant women in Ethiopia were included

Publication status: both published and unpublished articles were considered

Language: the articles published only in the English language were included

Publication year: all publications irrespective of publication or reporting year were considered (the published time was from 2014 to 2019)

2.4.2. Exclusion Criteria. Before exclusion, the titles, abstracts, and full texts of the studies were cautiously reviewed for eligibility. Articles that did not clearly report the prevalence of $\mathrm{HCV}$ among pregnant women in Ethiopia were excluded. In addition, articles published in languages other than English and outside Ethiopia were excluded. Review, case reports, editorials or commentaries, and case studies were also excluded.

2.5. Data Extraction. Data were extracted using a standardized data extraction tool adapted from Joanna Briggs Institute (JBI) Meta-Analysis of Statistics Assessment and Review Instruments [23]. All necessary data such as the name of the first author, year of study, year of publication, geographic region, study design, study setting, sample size, study population, serological evidence of $\mathrm{HCV}$, absolute numbers of HCV infected pregnant women, and prevalence of $\mathrm{HCV}$ were extracted. Then, all data were inserted into the data extraction template of Microsoft Office Excel 2013. 
2.6. Methodological Quality of Studies. The overall quality of all included studies was assessed using a critical appraisal checklist for observational studies adopted from JBI [23]. Full-text articles were reviewed for the following criteria: addressing the target population, adequacy of sample size, sampling methods, study population, data collection methods, definition of the variables and method of dealing with samples, data collection tools, statistical analysis tests, study objectives, illustration of the results, and presentation of the findings based on the objectives and adequacy of response rate. Each question was assigned one score with the possible range of scores being $0-8$. The JBI criteria for assessing the quality of primary studies recommend including primary studies that scored $\geq 60 \%$ of methodological checklists in the meta-analysis. Thus, all included studies were scored $\geq 80 \%$.

2.7. Outcome Measures. The main outcome required for the final meta-analysis was the "prevalence of HCV among pregnant women in Ethiopia." The prevalence was calculated by dividing the number of pregnant women with confirmed $\mathrm{HCV}$ infection by the total number of pregnant women who have been included in the study (sample size) multiplied by 100.

2.8. Data Synthesis and Analysis. Data were analyzed using statistical software STATA version 11 . To summarize the selected studies, tables and figures were used. The overall pooled prevalence of $\mathrm{HCV}$ among pregnant women was estimated using a random-effects meta-analysis. A randomeffects meta-analysis model was used to minimize the random variations between the point estimates of the primary studies since heterogeneity was exhibited. Point prevalence as well as their $95 \%$ confidence intervals (CI) was exemplified by forest plots. The degree of heterogeneity among the results of the selected studies was detected using the Cochrane test $(Q)$ and $I^{2}$ indicator [24]. To determine the possible reasons for substantial heterogeneity, subgroup analyses were conducted by geographical location (region) where the primary studies were conducted. Though the funnel plot and Egger's test are less reliable when the number of studies is less than ten, visual inspection of the asymmetry in funnel plots and Egger test were served to assess the presence of publication bias, with $p$ value less than 0.05 indicating significant publication bias [25].

\section{Results}

3.1. Study Selection. In the search of the literature, 502 potential studies were identified. Of these, 104 were duplicates and were removed. After screening the titles and abstracts, 347 irrelevant studies were excluded from the meta-analysis. Consequently, only 51 articles were considered for full-text review. After full-text review, 45 articles were removed: studies with no quantitative measures of hepatitis $\mathrm{C}$ virus in pregnant women; studies that were not conducted in Ethiopia; studies that provided combined $\mathrm{HCV}$ and hepatitis B virus prevalence; studies that did not meet the eligibility criteria. Finally, 6 articles were identified as eligible for meta-analysis. The overall selection process of studies was undertaken according to PRISMA flow diagram (Figure 1).

3.2. Study Characteristics. Five primary studies that fulfilled the eligibility criteria were institutional-based cross-sectional studies and one was community-based cross-sectional. The studies were conducted from 2013 to 2017 and published from 2014 to 2019 . The sample size among studies ranged from the smallest 222 [26] to the largest 455 [27]. The majority of the studies $(n=4)$ used random sampling methods whereas 2 studies used convenient sampling methods. The highest prevalence of HCV infection among pregnant women $(8.08 \%)$ was reported in East Wollega Zone [28] while the lowest prevalence $(0.26 \%)$ was reported from a study done in Felege Hiwot referral hospital [19]. Most of the included studies (4 out of 6) measured the prevalence of hepatitis $\mathrm{C}$ virus infection using the rapid test and the other 2 studies using the enzyme-linked immunosorbent assay (ELISA) test. After reviewing the methodological quality of the studies, 4 were deemed to be of good quality and 2 of medium quality, and no article was found with poor quality. Three regions of Ethiopia were represented in the included studies: three were conducted in Amhara [19, 29, 30], two were conducted in Oromia $[27,28]$, and one was conducted in SNNPR [26] (Table 1).

3.3. Quality Assessment. The quality of each study included in this systematic review and meta-analysis was critically evaluated using a critical appraisal checklist for observational studies adopted from the Joanna Briggs Institute (JBI). Four of the studies (66.7\%) scored $\geq 7$ "yes" out of 8 on the quality assessment scale which is $\geq 87.5 \%$ and determined to be of high quality. Two of the studies (33.3\%) were demeaned moderate quality as they scored 6 "yes" out of 8 on the quality scale assessment which is $75 \%$ (Table 2).

\subsection{Prevalence of HCV Infection among Pregnant Women.} In the current meta-analysis, a total of 2117 pregnant women were involved. The overall pooled prevalence of HCV infection among pregnant women in Ethiopia was $1.83 \%$ (95\% CI: $0.61,3.06)$. The $\mathrm{I}^{2}$ statistics for HCV infection among pregnant women was $I^{2}=87.4 \%(p<0.001)$ which indicates the presence of significant heterogeneity among the included studies. Hence, a random effect metaanalysis model was used to estimate the pooled prevalence of HCV infection among pregnant women in Ethiopia (Figure 2).

A metaregression analysis was done based on the categorical variables including sample size, year of publication, quality of included paper, and screening methods that primary studies have used. Accordingly, the analysis shows that all the variables included in the metaregression analysis had no significant effect on the pooled prevalence of HCV infection among pregnant women in Ethiopia (Table 3). 


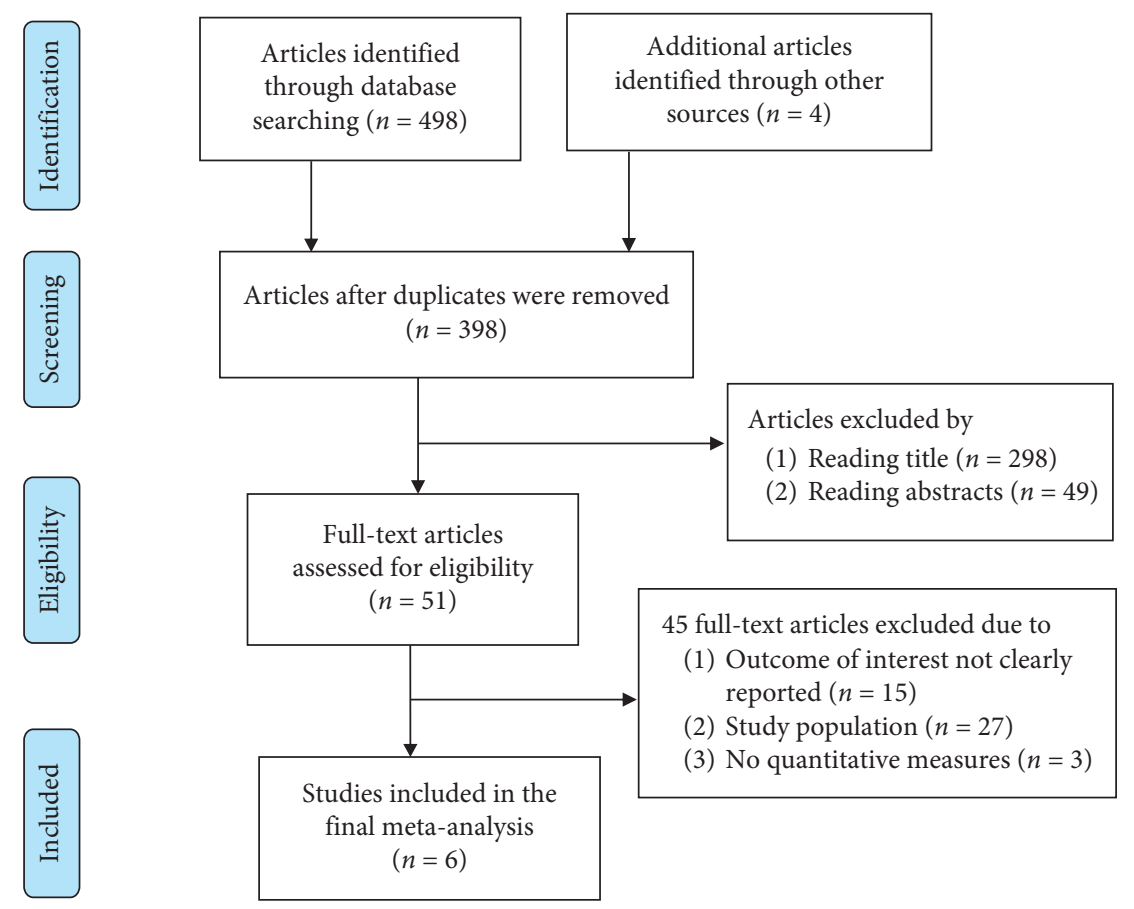

FIGURE 1: PRISA flow diagram of study selection for systematic review and meta-analysis of HCV infection among pregnant women in Ethiopia, 2020.

TABLE 1: Descriptive summary of primary studies included in the systematic review and meta-analysis of HCV prevalence among pregnant women in Ethiopia, 2020.

\begin{tabular}{|c|c|c|c|c|c|c|c|c|c|}
\hline First author & Year & Region & Study area & Study design & $\begin{array}{l}\text { Sample } \\
\text { size }\end{array}$ & Participants & Case & $\begin{array}{c}\text { Response rate } \\
(\%)\end{array}$ & $\begin{array}{c}\text { Prevalence } \\
(\%)\end{array}$ \\
\hline $\begin{array}{l}\text { Temesgen and } \\
\text { Andamlak }\end{array}$ & 2019 & SNNPR & Atat Hospital & Cross-sectional & 222 & 222 & 4 & 100 & 1.801802 \\
\hline Yohannes et al. & 2015 & Amhara & Bahir Dar city & Cross-sectional & 318 & 318 & 2 & 100 & 0.628931 \\
\hline Mohammed et al. & 2014 & Amhara & $\begin{array}{l}\text { Dessie referral } \\
\text { hospital }\end{array}$ & Cross-sectional & 385 & 385 & 3 & 100 & 0.779221 \\
\hline Sefinew et al. & 2015 & Amhara & $\begin{array}{l}\text { Felege Hiwot } \\
\text { referral hospital }\end{array}$ & Cross-sectional & 384 & 384 & 1 & 100 & 0.260417 \\
\hline Belayne et al. & 2019 & Oromia & Jimma & Cross-sectional & 455 & 387 & 9 & 87 & 2.325581 \\
\hline Regea and Eyasu & 2018 & Oromia & East Wollega zone & Cross-sectional & 422 & 421 & 34 & 99.8 & 8.07601 \\
\hline
\end{tabular}

TABle 2: Joanna Briggs Institute Critical Appraisal Checklist for analytical cross-sectional studies, 2020.

\begin{tabular}{|c|c|c|c|c|c|c|c|c|c|}
\hline Studies & $\begin{array}{c}\text { Clear } \\
\text { criteria } \\
\text { for } \\
\text { inclusion }\end{array}$ & $\begin{array}{c}\text { Detailed } \\
\text { description of } \\
\text { study subject } \\
\text { and setting }\end{array}$ & $\begin{array}{c}\text { Reliability } \\
\text { and validity } \\
\text { of study } \\
\text { tools }\end{array}$ & $\begin{array}{c}\text { Used } \\
\text { standard } \\
\text { criteria or } \\
\text { objective }\end{array}$ & $\begin{array}{l}\text { Identify } \\
\text { cofounding } \\
\text { factor }\end{array}$ & $\begin{array}{l}\text { Strategy } \\
\text { dealing } \\
\text { with } \\
\text { cofounders }\end{array}$ & $\begin{array}{c}\text { Outcome } \\
\text { measured } \\
\text { within a } \\
\text { valid way }\end{array}$ & $\begin{array}{l}\text { Appropriate } \\
\text { statistical } \\
\text { analysis used }\end{array}$ & $\begin{array}{c}\text { Overall } \\
\text { score } \\
(\%)\end{array}$ \\
\hline \multirow{6}{*}{$\begin{array}{l}\text { Temesgen and } \\
\text { Andamlak } \\
\text { Yohannes et al. } \\
\text { Mohammed } \\
\text { et al. } \\
\text { Sefinew et al. } \\
\text { Belayne et al. } \\
\text { Regea and } \\
\text { Eyasu }\end{array}$} & Yes & Yes & Yes & Yes & Yes & No & Yes & Yes & 87.5 \\
\hline & Yes & Yes & No & Yes & Yes & No & Yes & Yes & 75 \\
\hline & Yes & Yes & Yes & Yes & Yes & No & Yes & Yes & 87.5 \\
\hline & Yes & Yes & Yes & Yes & Yes & Yes & Yes & Yes & 100 \\
\hline & Yes & Yes & Yes & Yes & Yes & Yes & Yes & Yes & 100 \\
\hline & Yes & Yes & No & Yes & Yes & No & Yes & Yes & 75 \\
\hline
\end{tabular}

3.5. Subgroup Analysis. Since this meta-analysis exhibited considerable heterogeneity, subgroup analysis was done using regions (where the studies were conducted) to identify the possible sources of heterogeneity among the studies. The subgroup analysis indicated that the heterogeneity level was the highest among pregnant women $\left(I^{2}=92.9 \%\right)$ in Oromia 


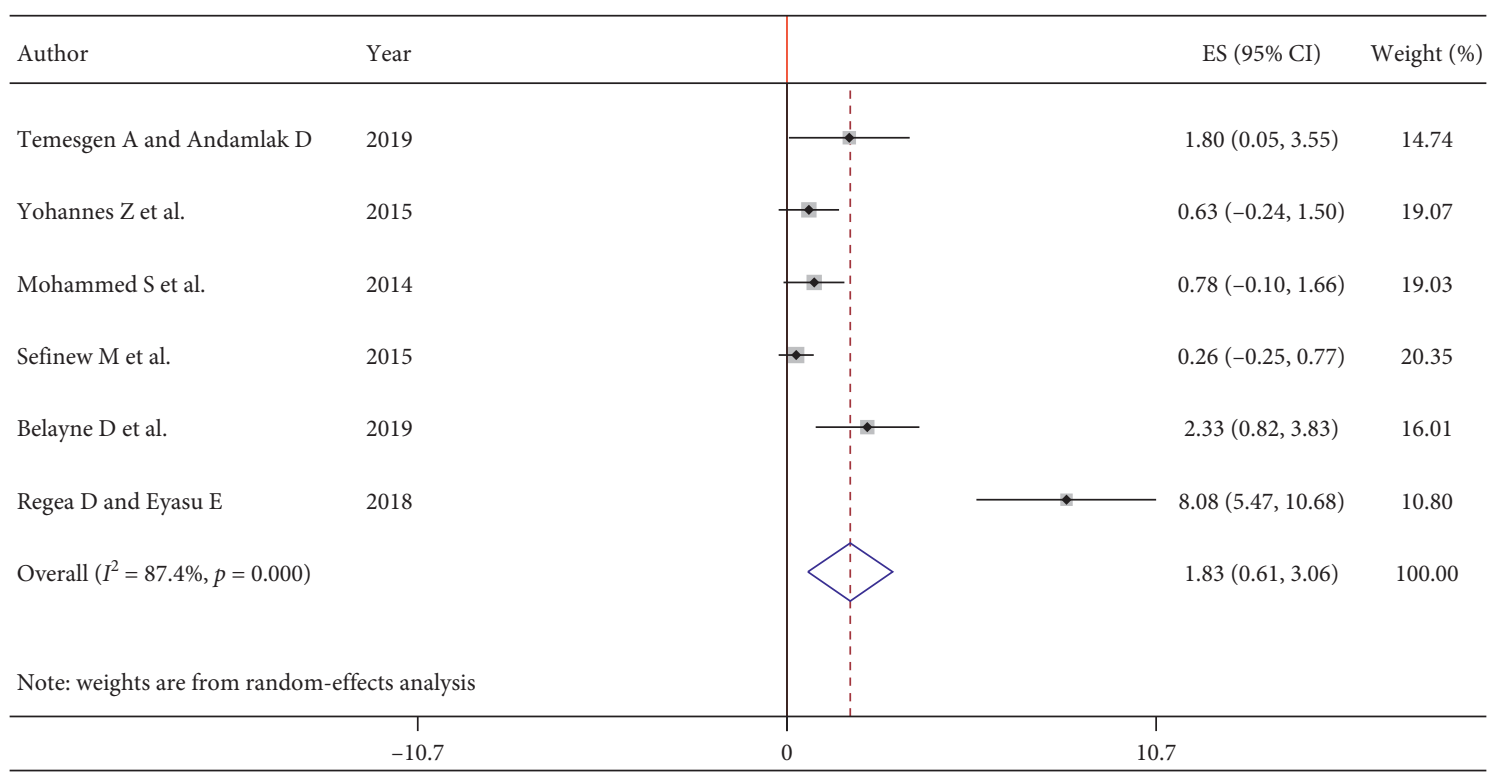

FIGURE 2: Forest plot of the pooled prevalence of HCV infection among pregnant women in Ethiopia, 2020.

TABLE 3: Results of bivariate metaregression for the prevalence of HCV infection among pregnant women in Ethiopia, 2020.

\begin{tabular}{lcccccc}
\hline Covariate & Category & Number of studies & Std. err. & Metaregression coefficient & $p$ value & Adjusted $R^{2}(\%)$ \\
\hline \multirow{2}{*}{ Year of publication } & Until 2015 & 3 & 2.683904 & 3.236623 & 0.154 & -29.99 \\
& After 2015 & 3 & 2.82759 & -2.683904 & 0.396 & 0.610 \\
\multirow{2}{*}{ Sample size } & $<384$ & 2 & 2.602811 & 1.438686 & -24.68 \\
& $>384$ & 4 & 4.501046 & -2443958 & 0.959 & 0.710 \\
\multirow{2}{*}{ Screening method used } & Rapid test & 4 & 2.645513 & -1.055988 & 0.396 & 0.46 \\
& ELISA & 2 & 3.760814 & 3.569947 & 0.317 & 0.686 \\
\multirow{2}{*}{ Quality of papers } & Good & 4 & 2.355038 & 2.69041 & 41.88 \\
\hline
\end{tabular}

region. Furthermore, the pooled estimate of subgroup analysis revealed that the prevalence of $\mathrm{HCV}$ among pregnant women was the highest $(5.10 \%)$ in Oromia region, compared with $0.44 \%$ in Amhara and $1.80 \%$ in SNNPR (Figure 3).

3.6. Publication Bias. Publication bias among the included studies for this meta-analysis was checked using visual inspection of the shape funnel plots and statistical Egger's test. The result of the tests revealed no evidence of publication bias according to Egger's test $(p=0.017)$ and symmetrical shape of funnel plots (Figure 4 ).

3.7. Discussion. The current systematic review and metaanalysis aimed to estimate the pooled prevalence of $\mathrm{HCV}$ infection among pregnant women in Ethiopia. The evidence obtained from this study may help healthcare workers and other concerned bodies to identify gaps in HCV screening during ANC; improve knowledge on the epidemiology of $\mathrm{HCV}$ infection among pregnant women in Ethiopia; and increase awareness of the general population regarding the epidemiology of $\mathrm{HCV}$.

In this systematic review and meta-analysis, the prevalence of $\mathrm{HCV}$ infection among pregnant women in Ethiopia is close to the WHO endemicity definition of $\mathrm{HCV}$ infection [7]. This finding implies, first, the need of evaluating the knowledge, skill, and readiness of healthcare workers on HCV screening of pregnant women during routine antenatal care. This may be attributed to the low awareness of the service provider at the service delivery level. Studies showed low awareness of hepatitis virus was reported in healthcare professionals $[16,19]$. Second, though the health system policy focuses on early detection of disease and complications during pregnancy as an element of focused antenatal care, this study indicates the need of evaluating its effectiveness. This may be attributed to less attention given to the health burden of viral hepatitis by the health system of Ethiopia. Third, due attention should be given to pregnant women so as to prevent the transmission of HCV to their newborns. Thus, screening of apparently healthy pregnant women can prevent the transmission and the complications $\operatorname{HCV}[8,31]$.

The overall pooled prevalence of $\mathrm{HCV}$ infection among pregnant women in Ethiopia was 1.83\% (95\% CI: 0.61, 3.06). Although there was no comparable meta-analysis study conducted on this specific research question, this finding is consistent with WHO intermediate definition of $\mathrm{HCV}$ infection (1.5\%-3.5\%) [7] and prevalence of HCV (3.1\%) in Ethiopia estimated by a systematic review and meta-analysis 


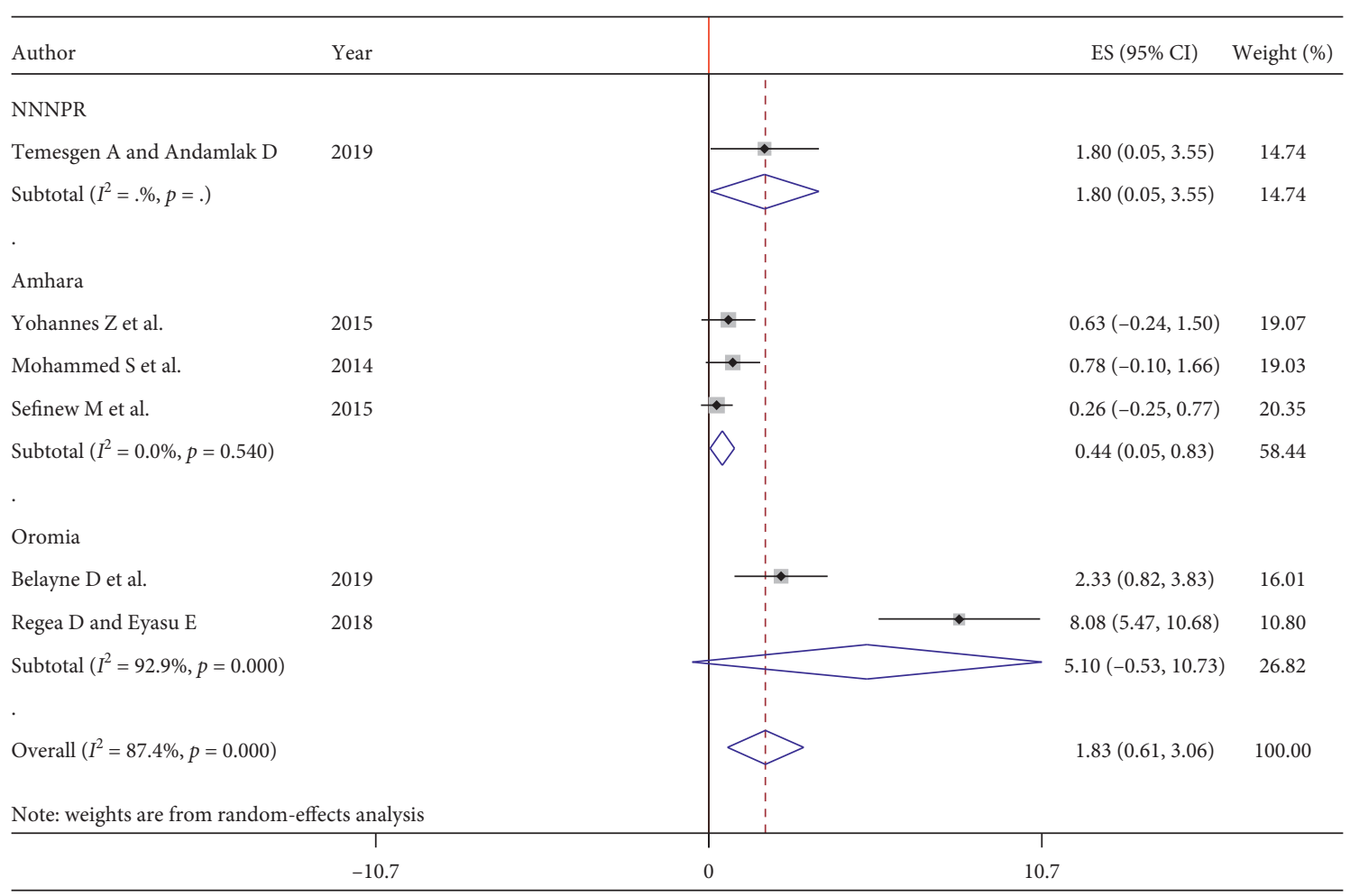

FIGURE 3: Subgroup analysis of HCV infection pooled prevalence estimation among pregnant women in Ethiopia, 2020.

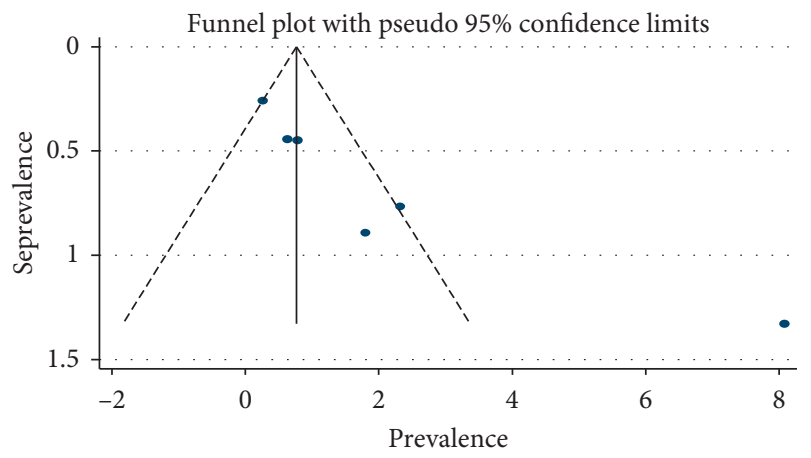

FIGURE 4: Funnel plot with pseudo 95\% confidence interval that investigated the publication bias of the pooled prevalence of $\mathrm{HCV}$ infection, Ethiopia, 2020.

conducted on hepatitis viruses in Ethiopia [17]. This may indicate the risk of $\mathrm{HCV}$ infection among pregnant women is comparable with the risk of the general population. The intermediate level of HCV infection should also act as a major alert for decision and policymakers in the Ethiopian health sector.

The present study covers three regions of Ethiopia. The result revealed that the prevalence of $\mathrm{HCV}$ among pregnant women was the highest (5.10\%) in Oromia region, compared with $0.44 \%$ in Amhara and $1.80 \%$ in SNNPR. This prevalence variation in different regions of Ethiopia could be due to differences in sample size, sampling method, and screening service and difference in the efficiency of diagnostic kits used. Moreover, the variation might be due to the difference in behavioral characteristics of the study participants including sexual practices, medical exposure for the potential risk factors of HCV infection, and level of awareness and differences in cultural practices.

3.8. Limitations of the Study. The prevalence estimates in this meta-analysis are likely to show the current situation of $\mathrm{HCV}$ infection among pregnant women in Ethiopia because the included studies were conducted in more recent years. The introduction of language bias is expected as articles published only in the English language were included. In addition, the heterogeneity across selected studies was high, but important sources of heterogeneity were not fully addressed. Subsequently, the question about the methodological rigor of the review may raise as the review process was conducted with a single author. Selection bias in the estimation of community prevalence may be introduced as many of the studies included in the meta-analysis recruited participants from the referral hospitals. Moreover, this metaanalysis represented only studies reported from three regions of Ethiopia, which could affect the estimated prevalence reported and its representatives.

\section{Conclusion}

This systematic review and meta-analysis confirmed the intermediate level of $\mathrm{HCV}$ infection among pregnant women in Ethiopia. The finding of this study suggests the need to implement a routine and universal HCV screening program for all pregnant women, which enables women to access $\mathrm{HCV}$ antiviral treatment to minimize vertical transmission to newborn infants. Moreover, national and 
regional health programs should mandate and monitor the screening procedures so as to reduce the risk of hepatitis $\mathrm{C}$ virus infection. Furthermore, increasing awareness on modes of transmission and prevention of HCV should be considered.

\section{Abbreviations}

ANC: Antenatal care

CI: $\quad$ Confidence interval

HCV: Hepatitis C virus

JBI: Joanna Briggs Institute

PRISMA: Preferred Reporting Items for Systematic Reviews and Meta-Analyses

SNNPR: Southern Nations Nationalities and People Region

WHO: World Health Organization.

\section{Data Availability}

All relevant data are included within the manuscript.

\section{Conflicts of Interest}

The author declares that there are no conflicts of interest.

\section{Authors' Contributions}

BDM designed the study; extracted, critically reviewed, and analyzed data; and wrote the manuscript. Finally, the author has read and approved the manuscript.

\section{Supplementary Materials}

Supplementary file 1: example of searches for the PubMed databases to assess the prevalence of HCV among pregnant women in Ethiopia. (Supplementary Materials)

\section{References}

[1] F. M. Averhoff, N. Glass, and D. Holtzman, "Global burden of hepatitis C: considerations for healthcare providers in the United States," Clinical Infectious Diseases, vol. 55, no. suppl_ 1, pp. S10-S15, 2012.

[2] W. H. Organization, Prevention and Control of Viral Hepatitis Infection: Framework for Global Action, World Health Organization, Geneva, Switzerland, 2012.

[3] S. Blach, S. Zeuzem, M. Manns et al., "Global prevalence and genotype distribution of hepatitis $C$ virus infection in 2015: a modelling study," The Lancet Gastroenterology \& Hepatology, vol. 2, no. 3, pp. 161-176, 2017.

[4] W. H. Organization, "Hepatitis C: Fact sheet No. 164," 2015, http://www.who.int/mediacentre/factsheets/fs164/en/.

[5] A. Schweitzer, J. Horn, R. T. Mikolajczyk, G. Krause, and J. J. Ott, "Estimations of worldwide prevalence of chronic hepatitis B virus infection: a systematic review of data published between 1965 and 2013," The Lancet, vol. 386, no. 10003, pp. 1546-1555, 2015.

[6] W. H. Organization, Global Hepatitis Report 2017, World Health Organization, Geneva, Switzerland, 2017.
[7] W. H. Organization, Global Policy Report on the Prevention and Control of Viral Hepatitis in WHO Member States, World Health Organization, Geneva, Switzerland, 2013.

[8] M. Arshad, S. S. El-Kamary, and R. Jhaveri, "Hepatitis C virus infection during pregnancy and the newborn period - are they opportunities for treatment?" Journal of Viral Hepatitis, vol. 18, no. 4, pp. 229-236, 2011.

[9] A. J. Esan, C. T. Omisakin, T. Ojo-Bola, M. F. Owoseni, K. A. Fasakin, and A. A. Ogunleye, "Sero-prevalence of hepatitis $\mathrm{B}$ and hepatitis $\mathrm{C}$ virue Co-infection among pregnant women in Nigeria," American Journal of Biomedical Research, vol. 2, no. 1, pp. 11-15, 2014.

[10] World Health Organization, World Health OrganizationHepatitis B Factsheet, World Health Organization, Geneva, Switzerland, 2017.

[11] K. L. B. Reddick, R. Jhaveri, M. Gandhi, A. H. James, and G. K. Swamy, "Pregnancy outcomes associated with viral hepatitis," Journal of Viral Hepatitis, vol. 18, no. 7, pp. e394-e8, 2011.

[12] A. Safir, A. Levy, E. Sikuler, and E. Sheiner, "Maternal hepatitis $B$ virus or hepatitis $C$ virus carrier status as an independent risk factor for adverse perinatal outcome," Liver International, vol. 30, no. 5, pp. 765-770, 2010.

[13] M. Gambarin-Gelwan, "Hepatitis B in pregnancy," Clinics in Liver Disease, vol. 11, no. 4, pp. 945-963, 2007.

[14] K. M. Zahran, M. S. Badary, M. N. Agban, and N. H. R. Abdel Aziz, "Pattern of hepatitis virus infection among pregnant women and their newborns at the Women's Health Center of Assiut University, Upper Egypt," International Journal of Gynecology \& Obstetrics, vol. 111, no. 2, pp. 171-174, 2010.

[15] L. E. Connell, H. M. Salihu, J. L. Salemi, E. M. August, H. Weldeselasse, and A. K. Mbah, "Maternal hepatitis B and hepatitis C carrier status and perinatal outcomes," Liver International, vol. 31, no. 8, pp. 1163-1170, 2011.

[16] F. Shiferaw, M. Letebo, and A. Bane, "Chronic viral hepatitis: policy, regulation, and strategies for its control and elimination in Ethiopia," BMC Public Health, vol. 16, no. 1, p. 769, 2016.

[17] Y. Belyhun, M. Maier, A. Mulu, E. Diro, and U. G. Liebert, "Hepatitis viruses in Ethiopia: a systematic review and metaanalysis," BMC Infectious Diseases, vol. 16, no. 1, p. 761, 2016.

[18] A. Bane, A. Patil, and M. Khatib, "Healthcare cost and access to care for viral hepatitis in Ethiopia," International Journal of Innovation and Applied Studies, vol. 9, no. 4, p. $1718,2014$.

[19] S. Molla, A. Munshea, and E. Nibret, "Seroprevalence of hepatitis B surface antigen and anti HCV antibody and its associated risk factors among pregnant women attending maternity ward of Felege Hiwot Referral Hospital, northwest Ethiopia: a cross-sectional study," Virology Journal, vol. 12, no. 1, p. 204, 2015.

[20] Division UNP, World Population Prospects: The 2019 Revision, Division UNP, New York, NY, USA, 2019, https://esaunorg/ unpd/wpp/.

[21] D. Moher, A. Liberati, J. Tetzlaff, D. G. Altman, and P. Group, "Reprint-preferred reporting items for systematic reviews and meta-analyses: the PRISMA statement," Physical Therapy, vol. 89, no. 9, pp. 873-880, 2009.

[22] V. Barbour, D. Moher, A. Liberati, J. Tetzlaff, and D. G. Altman, "Preferred reporting items for systematic reviews and meta-analyses: the PRISMA statement," PLoS Medicine, vol. 6, no. 7, Article ID e1000097, 2009. 
[23] J. B. Institute, “The Joanna briggs institute," Joanna Briggs Institute Reviewers' Manual: 2014 Edition, The Joanna Briggs Institute, Adelaide, Australia, 2014.

[24] T. B. Huedo-Medina, J. Sánchez-Meca, F. Marín-Martínez, and J. Botella, "Assessing heterogeneity in meta-analysis: Q statistic or $\mathrm{I}^{2}$ index?” Psychological Methods, vol. 11, no. 2, pp. 193-206, 2006.

[25] M. Egger, G. D. Smith, M. Schneider, and C. Minder, "Bias in meta-analysis detected by a simple, graphical test," British Medical Journal, vol. 315, no. 7109, pp. 629-634, 1997.

[26] T. A. Bafa and A. D. Egata, "Seroepidemiological patterns and predictors of hepatitis B, C and HIV viruses among pregnant women attending antenatal care clinic of Atat Hospital, Southern Ethiopia," SAGE Open Medicine, vol. 8, 2020.

[27] B. D. Taye, R. Kedir, L. Teshager et al., "Prevalence and associated risk factors of hepatitis $\mathrm{B}$ and $\mathrm{C}$ virus infections among mothers in Jimma town, South West Ethiopia: a community based study," Ethiopian Medical Journal, vol. 57, 2019.

[28] R. Dabsu and E. Ejeta, "Seroepidemiology of hepatitis B and C virus infections among pregnant women attending antenatal Clinic in Selected Health Facilities in east Wollega zone, West Oromia, Ethiopia," BioMed Research International, vol. 2018, Article ID 4792584, 9 pages, 2018.

[29] M. Seid, B. Gelaw, and A. Assefa, "Sero-prevalence of HBV and HCV infections among pregnant women attending antenatal care clinic at Dessie Referral Hospital, Ethiopia," Advance Life Science Health, vol. 1, no. 2, pp. 109-120, 2014.

[30] Y. Zenebe, W. Mulu, M. Yimer, and B. Abera, "Sero-prevalence and risk factors of hepatitis $\mathrm{C}$ virus infection among pregnant women in Bahir Dar city, Northwest Ethiopia: cross sectional study," Pan African Medical Journal, vol. 21, no. 1, 2015.

[31] T. Manyazewal, Z. Sisay, S. Biadgilign, and W. E. Abegaz, "Hepatitis B and hepatitis C virus infections among antiretroviral-naive and-experienced HIV co-infected adults," Journal of Medical Microbiology, vol. 63, no. 5, pp. 742-747, 2014. 